\title{
High-precision absolute laser interferometer distance measurement system
}

\author{
Chen-yang Zhang,Shao-lan Zhu,Hao-dong He and \\ Bing Li \\ Xi'an Institute of Optics and Precision Mechanics of \\ Chinese Academy of Sciences \\ Xi'an, 710119, China \\ zhangchenyang@opt.cn
}

\author{
Chen-yang Zhang,Shao-lan Zhu and Bing Li \\ Garduate University \\ Chinese Academy of Sciences \\ Beijing, 10039,China \\ zhangchenyang@opt.cn
}

\begin{abstract}
With the improvement of precision in various fields, we increase high requirements for measurement accuracy. In this paper, we obtain the interference fringes change information (the distance information) with the help of laser scanning with different frequency. This interference method is an absolute measurement. It does not require the target to move in the direction of measurement. In this paper, we give a detailed analysis of the theory. We have done experiments to compare this new method's results with the results of RENISHAW interferometer. It's improved that the accuracy of distance measurement is $10-4 \sim 10-5$ relatively.
\end{abstract}

Keywords-Absolute distance metrology, high-precision, Tunable laser, Interferometer, Subdivision

\section{INTRODUCTION}

Laser ranging techniques are widely used to perform contactless measurement of the distance of a remote target for both industrial and scientific applications. These techniques are useful when contact with the target is not possible, the target is surrounded by a hostile environment, and/or no perturbation on the target is to be made. Commercially available instruments are based either on TOF methods(telemeters), which are best suited for long-range applications, or on triangulation techniques, which better apply to short-range measurements[3].We choose the technique of interferometer in the distance measurement system for its non-contact, high-precision and highsensitivity. In traditional incremental interferometry, we change the optical path difference by adjust the geometric distance. The different optical path length brings changes to both of the phase of the interference optical path and the stripes. This method needs high demands on the optical path in the measurement process and it must be a moving target mirror during the measurement. Unlike traditional interference method, this system is independent of the intermediate process of the length and it does not have to move any parts. It uses the characteristics of the semiconductor laser that its frequency is changed with the change of injection current and temperature in a function relation. The phase of interferential light is changed when the laser frequency (wavelength) is changing. Then we get the distance information by the changed information of the interference fringes.

\section{METHODOLOGY}

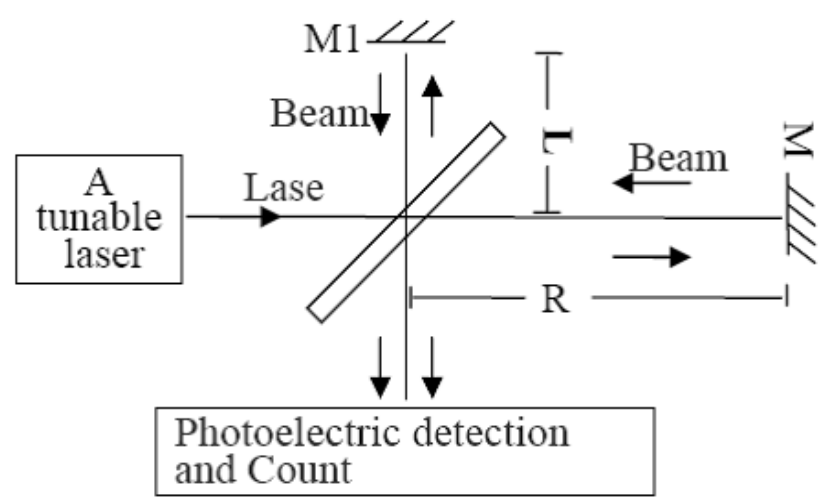

Fig.1:Typical Michelson-like interferometer

A typical Michelson-like interferometer is shown in Fig.1. These are generally based on Michelson interferometers that are read-out using a laser source with continuously tuneable emission wavelength. Wavelength sweeping generates an interferometric signal, that carries information about the difference in length of the two interferometer arms[1].In traditional incremental interferometry, one arm is fixed, the other arm is moved during the process of distance measurement. Then we obtain distance information. Since this method requires an external modulator, it generates rather large cyclic error that originates from electrical and optical crosstalk. It also requires the target mirror continuously moving from the agreed zero point to test point and it does not allow light to depart[5],[6]. The advantage of the paper's method lies in the measurement process without having to move any arm. We only care about the starting point and the endpoint of the measurement. If you pre-set the zero position, we only need to directly place the target mirror in the test point during the process of measurements. The precondition of this new method is that there is no change of the optical path difference between the arms. Then we make a linear change of the laser output frequency (wavelength), and the phase of the interference and the interferential fringes are changed. We count the integer and fractional fringe number and get the distance. 


\section{DEMONSTRATE}

High-precision absolute long distance measurements are essential for application to a wide range of fields such as industrial sensing and positioning in space. Conventionally, phase measurements of a sinusoidally modulated CW optical wave have been carried out for long-distance measurements. Since this method requires an external modulator, it generates rather large cyclic error that originates from electrical and optical crosstalk[4].

Then, It is possible to express the measured phase difference $\varphi_{1}$ in terms of the initial output frequency of the $\operatorname{laserv}_{1}$ :

$$
\varphi_{1}=\frac{4 \pi n v_{1}}{C}(R-L)
$$

If $v_{1}$ becomes $v_{2}$, then the measured phase difference $\varphi_{2}$ is:

$$
\varphi_{2}=\frac{4 \pi n v_{2}}{C}(R-L)
$$

When the frequency changes fromv $v_{1}$ tov $_{2}$, the change of the measured phase difference is $\Delta \varphi$ :

$$
\Delta \varphi=\varphi_{2}-\varphi_{1}=\frac{4 \pi n \Delta v}{c}(R-L)
$$

Now, the variation of stripe is $\mathrm{N}$ :

$$
\Delta \varphi=2 \pi \mathrm{N}
$$

If the point of $\mathrm{R}=\mathrm{L}$ is the starting point (the point $\mathrm{O}$ ) and the endpoint is $\mathrm{A}$, then the distance is OA:

$$
\mathrm{OA}=\left(\mathrm{R}_{\mathrm{A}}-\mathrm{L}\right)-(\mathrm{R}-\mathrm{L})=\left(\mathrm{R}_{\mathrm{A}}-\mathrm{L}\right)=\frac{C}{2 \pi \Delta v} N_{A}
$$

\section{SYSTEM DESIGN}

Schematic overview of absolute interferometric measurement system is shown in Fig.2. In all of these applications of radar interferometry, the light emitted by a laser diode is back-scattered by a remote target and reenlters the laser cavity, thus generating an interferometric signal. In the distance easurement approach, the laser wavelength is linearly modulated, and the target distance is estimated from the count of the number of interferometric fringes. The ability of the system to measure phase and amplitude between two channels is one of the driving components that sets the accuracy of the final measurement.

Thus, We can get the two signals ( $\sin$ and cos) by shifting the phase after extracting the stripes' change, and get the subdivision of the wavelength average which is divided into eight regions with two-way signal amplitude as a benchmark. We increase the resolution to the eighth of the wavelength in this way. Fig. 3 and Fig. 4 mimics this process.

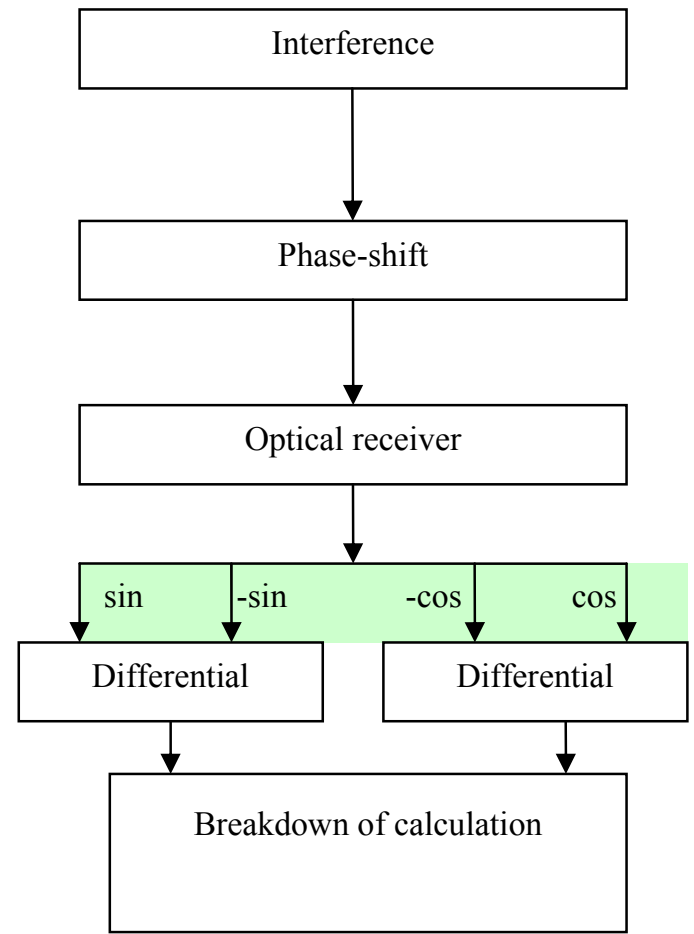

Fig.2:Schematic overview of absolute interferometric measurement system

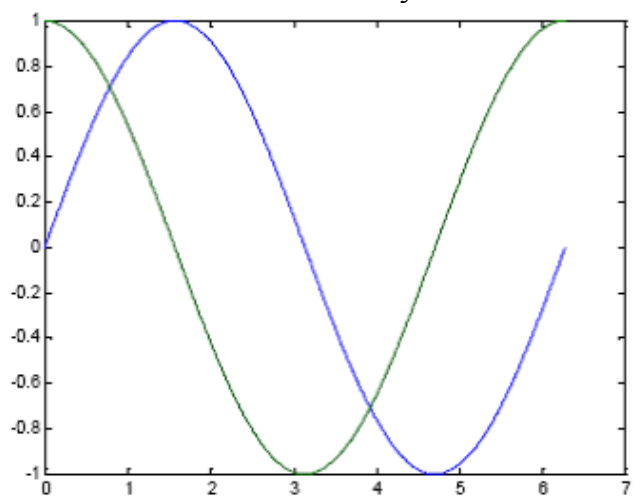

Fig.3:Sin and cos signals received

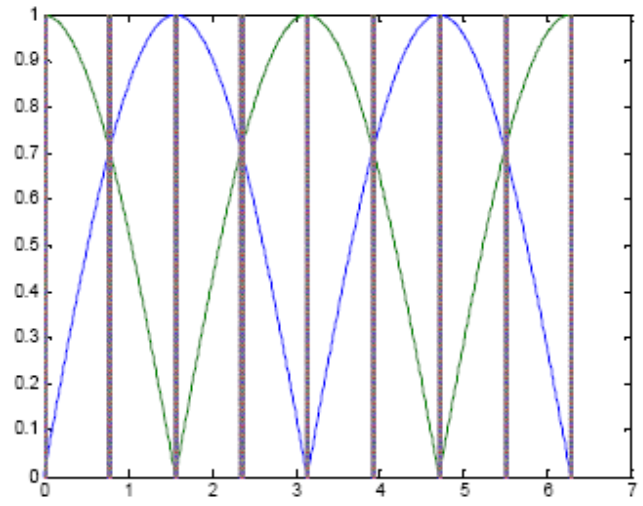

Fig.4:Segments of the sin and cos signals 
The table 1 provided the corresponding subdivided interval. Then final measurement result is the integer number with the addition of the fractional fringe number. In theory, this segmentation method can obtain high-precision distance information, but it requires a higher anti-jamming capability. Indeed, the relationship between observing errors and system parameters plays a fundamental role in the design of observing strategies for science applications such as weather radar, synthetic aperture radar, and interferometric synthetic aperture radar where wideband signals resulting from multiple sources of reflection are superimposed on one another[2]. The displacement measuring method based on laser interference principle obtains measured displacement by processing interference fringes' movement and is widely used in precision measurement. Usually two difference signals are used to discerning direction, count and subdivision in laser interferometry system.

Table 1:Subdivided interval corresponding table

\begin{tabular}{|c|c|}
\hline Sin and cos signal comparison & Subdivided interval \\
\hline $0<\sin <\cos$ & $1 / 8$ Wavelength \\
\hline $0<\cos <\sin$ & $2 / 8$ Wavelength \\
\hline $\operatorname{Cos}<0<\sin -\cos <\sin$ & $3 / 8$ Wavelength \\
\hline $\operatorname{Cos}<0<\sin -\cos <\sin$ & $4 / 8$ Wavelength \\
\hline $\operatorname{Cos}<\sin <0$ & $5 / 8$ Wavelength \\
\hline $\operatorname{Sin}<\cos <0$ & $6 / 8$ Wavelength \\
\hline $\operatorname{Sin}<0<\cos \cos <$-sin & $7 / 8$ Wavelength \\
\hline $\operatorname{Sin}<0<\cos \cos >$-sin & 1 Wavelength \\
\hline
\end{tabular}

\section{MEASUREMENT RESULTS}

In the experiment, the system and the British TENISHAW interferometer. X-80 were compared on the rail. The experimental results are shown in Table 2:

Table 2:The experimental results

\begin{tabular}{|c|c|c|c|}
\hline \multicolumn{4}{|c|}{ Results (mm) } \\
\hline $\begin{array}{c}\text { RENISHAW } \\
\text { X-80 }\end{array}$ & 800.0522 & 800.3614 & 800.07499 \\
\hline Our system & 800.0617 & 800.3714 & 800.0845 \\
\hline $\begin{array}{c}\text { RENISHAW } \\
\text { X-80 }\end{array}$ & 800.7987 & 800.5627 & 800.07499 \\
\hline Our system & 800.8077 & 800.5715 & 800.0845 \\
\hline
\end{tabular}

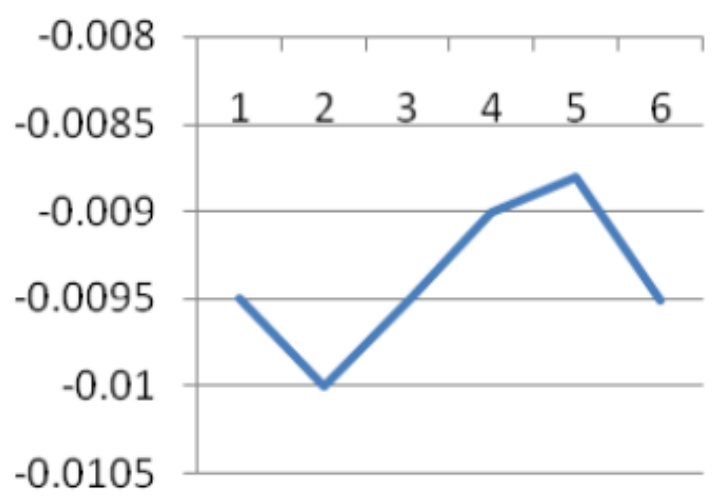

Fig.5:Error curve

Error curve is shown in Fig.5. The laser source with a continuously tunable emission wavelength is used to read out an interferometer, and the obtained interferometric signal carries information about the difference in length of the reference and the measuring arms of the interferometer. The error is introduced by the relative stability of the light source. After the amendments, the relative length measurement accuracy can be increased to 10-4 10-5, but at the expense of a complicated experimental setup, which often requires a interferometer for calibration.

\section{CONCLUSIONS}

The change of laser frequency causes the change of stripes throughput. In this paper, we change the traditional incremental interferometer into absolute interference. This approach eliminates the impact of the move process and improves the accuracy of the static measurement characteristics. The anti-jamming capability of the system is enhanced and the measurement accuracy is improved by stripes subdivision.

\section{REFERENCES}

[1] Michele Norgia, Guido Giuliani and Silvano Donati,"New Absolute Distance Measurement Technique by Self-Mixing Interferometry in Closed Loop" Instrumentation and Measurement Technology Conference Como, Italy,2004.

[2] Michele Norgia, Guido Giuliani and Silvano Donati,"Absolute Distance Measurement With Improved Accuracy Using Laser Diode Self-Mixing Interferometry in a Closed Loop" IEEE transactions on Instrumentation and Measurement, vol.56,2007

[3] Paul Siqueira, Razi Ahmed, John W. Wirth and Alex Bachmann, "Variable Precision Two-Channel Phase, Amplitude, and Timing Measurement for Radar Interferometry and Polarimetry" IEEE transactions on microwave theory and techniques, vol.55,2007.

[4] K.Minoshima "High-precision Absolute Length Metrology using Fiber-Based Optical Frequency Combs”IEEE,2010.

[5] D.Xiaoli and S. Katuo, "High-accuracy absolute distance measurement by means of wavelength scanning heterodyne interferometry",Meas.Sci.Technol.9,1031-1035(1998).

[6] B. Calvel, I. Cabeza, A. Cabral, E. Manske, J. Rebordao, R. Sesselmann, Z. Sodnik, A.Verlaan, "High Precision Optical Metrology for Darwin: Design and Performance", $6^{\text {th }}$ International Conference on Space Optics, Toulouse, 2004 